\title{
A Recombinant Rotavirus Antigen Based on the Coat Protein of Alternanthera Mosaic Virus
}

\author{
E. M. Ryabchevskaya ${ }^{a}{ }^{*}$, E. A. Evtushenko ${ }^{a}$, M. V. Arkhipenko ${ }^{a}$, E. K. Donchenko ${ }^{a}$, \\ N. A. Nikitin ${ }^{a}$, J. G. Atabekov ${ }^{a, b}$, and O. V. Karpova ${ }^{a}$ \\ ${ }^{a}$ Department of Virology, Faculty of Biology, Moscow State University, Moscow, 119234 Russia \\ ${ }^{b}$ Institute of Bioengineering, Federal Research Center "Fundamentals of Biotechnology", Russian Academy of Sciences, \\ Moscow, 117312 Russia \\ *e-mail: eryabchevskaya@gmail.com
}

Received July 17, 2019; revised August 30, 2019; accepted September 2, 2019

\begin{abstract}
Thanks to their strong immunostimulating properties and safety for humans, plant viruses represent an appropriate basis for the design of novel vaccines. The coat protein of Alternanthera mosaic virus can form virus-like particles that are stable under physiological conditions and have adjuvant properties. This work presents a recombinant human rotavirus A antigen based on the epitope of rotavirus structural protein VP6, using Alternanthera mosaic virus coat protein as a carrier. An expression vector containing the gene of Alternanthera mosaic virus (MU strain) coat protein fused to the epitope of rotavirus protein VP6 was designed. Immunoblot analysis showed that the chimeric protein was effectively recognized by commercial polyclonal antibodies to rotavirus and therefore is a suitable candidate for development of a vaccine prototype. Interaction of the chimeric recombinant protein with the native coat protein of Alternanthera mosaic virus and its RNA resulted in the formation of ribonucleoprotein complexes that were recognized by antirotavirus antibodies.
\end{abstract}

Keywords: plant viruses, vaccines, rotavirus infection, virus-like particles, potexvirus

DOI: $10.1134 / \mathrm{S} 0026893320020132$

\section{INTRODUCTION}

Rotavirus infection is one of a major causes of acute gastroenteritis in children under 5 years of age, leading annually to the death of nearly 215000 children worldwide [1]. Furthermore, rotavirus can also be dangerous for patients of other age groups. Over $90 \%$ of all infection cases are caused by rotavirus A strains [2]. The great serological diversity of rotavirus A strains is a serious problem for vaccine development. The currently existing vaccines against rotavirus infection are live attenuated vaccines for peroral administration. However, they have certain shortcomings, such as the risk of reversion to pathogenicity or the appearance of novel vaccine-associated reassortants of the virus, as well as the risk of intussusception (a serious complication caused by peroral vaccine administration) [3, 4]. In this context, development of a recombinant vaccine against rotavirus $A$ that could be administered perorally is a topical issue.

Structural proteins of rotavirus form a three-layered capsid. The inner layer is made of VP2 dimers, the intermediate layer is made of VP6 trimers, and the

Abbreviations: AltMV, Alternanthera mosaic virus; CP, coat protein; VLP, virus-like particle; RNP, ribonucleoprotein. outer layer is composed of VP7 trimers and VP4 trimers, which form spikes. For the virus to enter a host cell, VP4 has to undergo proteolysis, producing VP5* and VP8* fragments [5]. The major rotavirus antigens are VP6, VP7, VP5*, and VP8*. The available data indicate that the structural protein VP6 is highly conserved: $87 \%$ of its amino acid sequence is invariable in group A strains [6]. VP6 plays a central role in the development of the specific immune response: the major antibodies produced in response to a natural rotavirus infection are specific to VP6. It was shown that the RLSFQLMRPPNMTP epitope of VP6 can activate a strong protective immune response comparable to the response induced by immunization with full-size VP6 [7]. For this reason, this epitope can be considered a promising candidate to be included in recombinant vaccines against rotavirus.

There exist different strategies for the development of recombinant vaccines. For anti-rotavirus vaccines, the major approaches are recombinant subunit vaccines containing individual rotavirus proteins, and vaccines based on virus-like particles (VLPs) composed of rotavirus proteins or proteins of other viruses [3]. In particular, it seems promising to obtain VLPs based on coat proteins of plant viruses, which are safe 
for humans, possess strong immunostimulating properties, and can be obtained cost-effectively [8]. One plant virus with immunostimulating properties is Alternanthera mosaic virus (AltMV) [9]. AltMV belongs to the Potexvirus genus of the Alphaflexiviridae family. AltMV virions have a filamentous shape, being $570 \mathrm{~nm}$ long and $13.5 \mathrm{~nm}$ in diameter [10]. The AltMV Coat protein (CP) is composed of 207 amino acid residues and has a molecular weight of $22 \mathrm{kDa}$; it can form filamentous virus-like particles (VLPs) in vitro in absence of RNA. In our previous work [11], it was shown that these VLPs, similarly to AltMV virions, exhibit adjuvant properties. AltMV VLPs are $15.2 \mathrm{~nm}$ in diameter, range in length from 60 to $2000 \mathrm{~nm}$, and are stable under physiological conditions [10, 12]. Thus, AltMV CP can be considered a promising carrier of foreign epitopes (antigenic determinants) for the development of recombinant vaccines.

The goal of this work was to obtain a recombinant rotavirus A antigen based on the epitope of VP6 with AltMV CP as the carrier, and to study the prospects of employing it as a component of a vaccine candidate against rotavirus $\mathrm{A}$.

\section{EXPERIMENTAL}

Isolation of AltMV, viral RNA, and coat protein. A preparation of AltMV (strain MU) was obtained from infected tobacco plants (Nicotiana benthamiana) [10]. RNA was isolated by phenol deproteinization; AltMV $\mathrm{CP}$ was isolated by salt deproteinization with $2 \mathrm{M} \mathrm{LiCl}$.

Obtaining AltMV CP fused to the epitope of VP6. The nucleotide sequence encoding recombinant AltMV CP fused to the epitope of rotavirus protein VP6 was obtained using a template of a plasmid carrying a cDNA copy of the AltMV genome (strain MU) (GenBank: FJ822136.1) [13]. The nucleotide sequence encoding AltMV CP was optimized for expression in a bacterial system; for this purpose, codons rarely occurring in Escherichia coli were replaced with those typical for these cells (https://www.genscript.com/ tools/codon-frequency-table). The forward primer 5'-ATACTCATGAGTACTCCATTTCCTCAAGTCACCCAGGAACAGATTGAC-3' containing a PagI recognition site and the reverse primer 5'-GTATGGATCCCGGCGTCATGTTCGGCGGGCGCATCAGTTGGAATGACAAACGCTCCGGTGGTGGGAGGTATTG-3', which contained the nucleotide sequence encoding the epitope of VP6 and a BamHI site, were utilized to obtain a nucleotide sequence encoding AltMV CP fused to the rotaviral epitope at the C-terminus. All genetic engineering procedures with recombinant DNA were performed using conventional techniques [14]. Recombinant DNA encoding AltMV CP fused to the epitope of VP6 was cleaved with PagI and BamHI restriction endonucleases (Thermo Fisher Scientific, United States) at the corresponding sites and ligated into a pQE-60 vector (QIAGEN) with T4 DNA ligase (Fermentas, Lithua- nia). The obtained plasmid was used to transform E. coli cells of the SG[pREP4] strain. After the expression was induced, recombinant protein was purified using affinity chromatography on Ni-NTA agarose (Qiagen, Germany) with $6 \mathrm{M}$ guanidine chloride and $8 \mathrm{M}$ urea as described in a handbook [15]. The preparation was dialyzed against Milli-Q deionized water (Merck Millipore, United States) exchanged hourly for $4 \mathrm{~h}$.

Immunoblotting. Proteins were separated by SDSPAGE in an $8-20 \%$ gradient gel and transferred onto a Hybond-P polyvinylidene fluoride membrane (Amersham, United States) using a Pierce Power Blotter electric transfer system (Thermo Scientific). Ribonucleoprotein complexes (RNPs) were analyzed by electrophoresis in a $1 \%$ agarose gel and transferred onto Hybond-P membranes using wet transfer in a desiccator overnight at room temperature. In both cases, the membranes were next treated with primary polyclonal goat antibodies to rotavirus A (ab31435, Abcam, Great Britain) at a $1: 1500$ dilution and then with secondary antibodies to goat IgG conjugated with horseradish peroxidase (Promega, United States) in a $1: 10000$ dilution. The membranes were treated with ECL substrate (Amersham), and the chemiluminescence signals were registered using the ChemiDoc XRS+ system (Bio-Rad, United States).

Incubation of RNA with proteins. RNA was incubated with proteins at a mass proportion of $1: 7$ or $1: 14$ in $20 \mu \mathrm{L}$ of $0.01 \mathrm{M}$ citrate buffer ( $\mathrm{pH} 4.0$ ) optimal for AltMV CP polymerization [12] for $20 \mathrm{~min}$ at room temperature.

Electron microscopy. The preparations were loaded on copper mesh grids for electron microscopy covered with carbon-coated collodion support films as described in other work [16] and analyzed using a JEOL JEM-1400 transmission electron microscope (JEOL, Japan) at a voltage of $80 \mathrm{kV}$; images were registered with an Olympus Quemesa digital camera using the iTEM software (Olympus Soft Imaging Solutions GmbH, Germany).

\section{RESULTS}

\section{Recombinant AltMV CP Fused to a Rotavirus A Epitope}

We obtained a recombinant protein composed of AltMV CP (strain MU) fused at the C-terminus to the RV14 epitope of rotavirus A protein VP6 (amino acid residues 289-302, RLSFQLMRPPNMTP); it was termed ER6 (Epitope of Rotavirus protein VP므). The schematic structure of ER6 is presented in Fig. 1a. Electrophoretic analysis of the ER6 preparation revealed a band with mobility corresponding to a molecular mass of $25 \mathrm{kDa}$ (Fig. 1b, lane 2), which was consistent with the expected value calculated using the ProtParam software (http://www.expasy.org). Immunoblotting confirmed that the protein contained amino acid sequence fragments recognized by anti- 
(a)

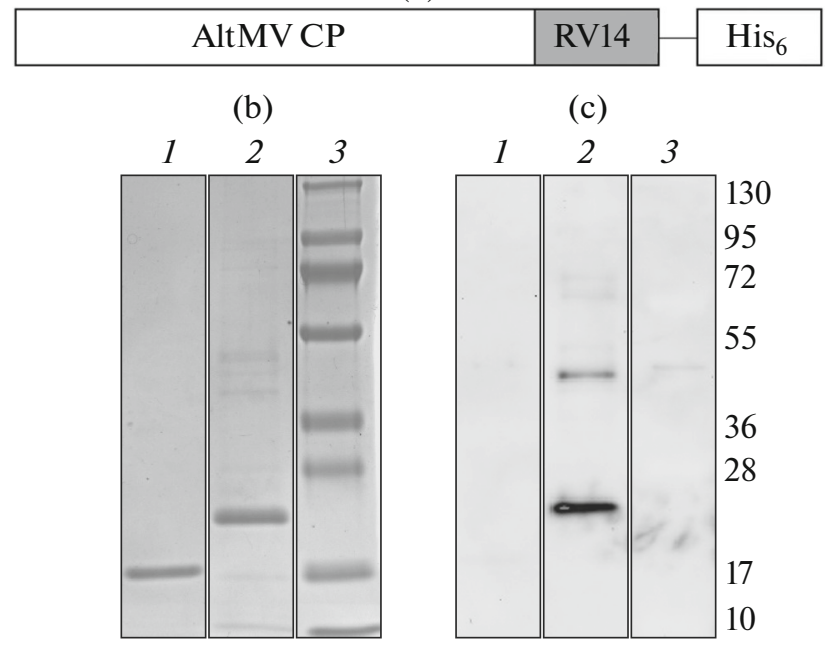

Fig. 1. ER6, a recombinant protein containing an epitope of rotavirus A. (a) Schematic structure of the recombinant protein ER6: RV14, an epitope of VP6 protein of rotavirus A (RLSFQLMRPPNMTP); His $_{6}$, a sequence of six histidine residues. (b) Analysis of ER6 by SDS-PAGE in an 8$20 \%$ gradient gel; staining with Coomassie G-250. (c) Immunoblot of recombinant protein ER6 with antibodies to rotavirus A. (b and c) (1) Tobacco mosaic virus (negative control); (2) ER6; (3) molecular mass markers (Thermo Scientific); molecular mass values are given in $\mathrm{kDa}$. Primary antibodies were commercial polyclonal antibodies to rotavirus A. Secondary antibodies were species-specific antibodies conjugated with horseradish peroxidase.

bodies to rotavirus A (Fig. 1c, lane 2). The ER6 preparation contained an admixture of protein with a higher molecular weight that also interacted with these antibodies and therefore probably represented a multimeric form of ER6.

\section{RNPs Containing ER6 and AltMV CP}

AltMV RNA was incubated with proteins in a proportion of $1: 7$ or $1: 14$, as proposed in similar experiments with another member of the Potexvirus family: potato virus $X[17,18]$. Incubation of proteins and viral RNA was performed in the following variants: $1 \mu \mathrm{g}$ RNA $+7 \mu \mathrm{g}$ native AltMV CP isolated form the virus (Fig. 2, lane 2); $1 \mu \mathrm{g}$ RNA $+7 \mu \mathrm{g}$ native AltMV CP + $7 \mu \mathrm{g}$ recombinant ER6 (Fig. 2, lane 3); $1 \mu \mathrm{g}$ RNA + $7 \mu \mathrm{g}$ recombinant ER6 (Fig. 2, lane 4). The control sample contained $1 \mu \mathrm{g}$ viral RNA incubated in the absence of protein under the same conditions (Fig. 2, lane 1).

Using a gel retardation assay, it was shown that incubation of RNA with two proteins simultaneously, AltMV CP and recombinant ER6, resulted in canonical RNP formation: the band corresponding to free RNA disappeared, and products with greater molecular weights corresponding to RNPs of different sizes appeared (Fig. 2, lane 3). It should be noted that this mixture also contained RNA that remained at the start. In experiments where RNA was mixed with a

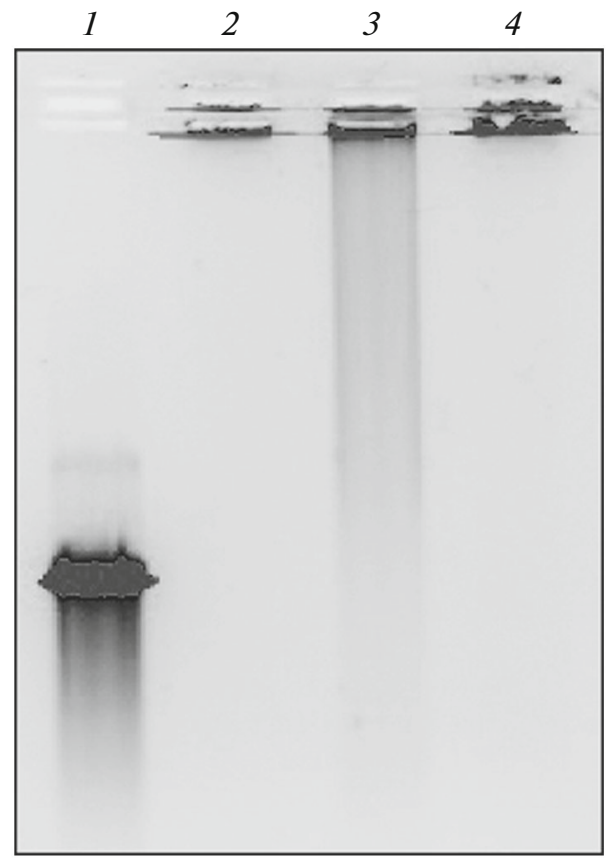

Fig. 2. Analysis of RNPs using gel retardation assay. (1) AltMV RNA (control); (2) AltMV RNA + AltMV CP (1:7); (3) AltMV RNA + AltMV CP + ER6 (1: $7: 7)$; (4) AltMV RNA + ER6 (1:7). Electrophoresis in a 1\% agarose gel; ethidium bromide staining. The proportion between viral RNA and proteins (AltMV CP and/or ER6) is indicated in parentheses.

single protein, either native AltMV CP or recombinant ER6 (Fig. 2, lanes 2 and 4, respectively), all RNA was retained at the start.

\section{Morphology of the Obtained RNPs}

Transmission electron microscopy was used to analyze the products of AltMV RNA interaction with AltMV CP (Fig. 3c) and ER6 (Figs. 3d, 3e) individually, and with a mixture of AltMV CP and ER6 (Fig. 3f). Preparations of AltMV CP and ER6 incubated under conditions of RNP formation ( $0.01 \mathrm{M}$ citrate buffer, $\mathrm{pH}$ 4.0) in the absence of RNA served as controls (Figs. $3 a$ and $3 b$, respectively). It was found that under these conditions, AltMV CP formed filamentous VLPs that were longer than AltMV virions [10] (Fig. 3a). The morphology of these VLPs did not change in the presence of RNA (Fig. 3c).

In contrast, ER6 was found to form aggregates, both if incubated individually (Fig. 3b) and with RNA (Fig. 3d). However, in samples of RNA with ER6 were rarely detected aggregates with a morphology resembling VLP conglomerates (Fig. 3e).

In the preparation where AltMV RNA was incubated with a mixture of AltMV CP and ER6, the pattern of RNP assembly was different: there were particles of varying length and with a morphology that visually resembled AltMV virions (Fig. 3f). These data, 


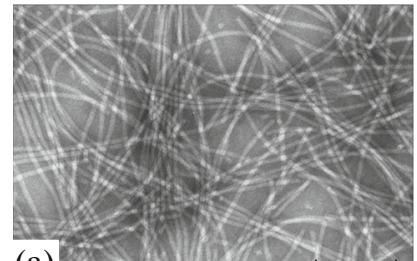

(a)
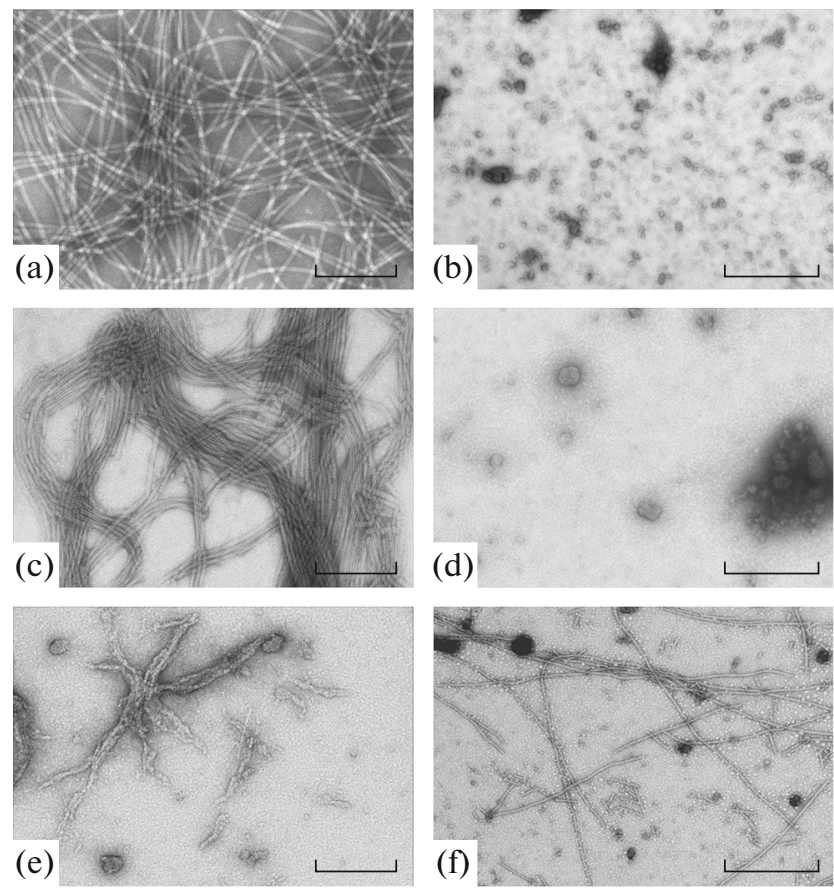

(d)
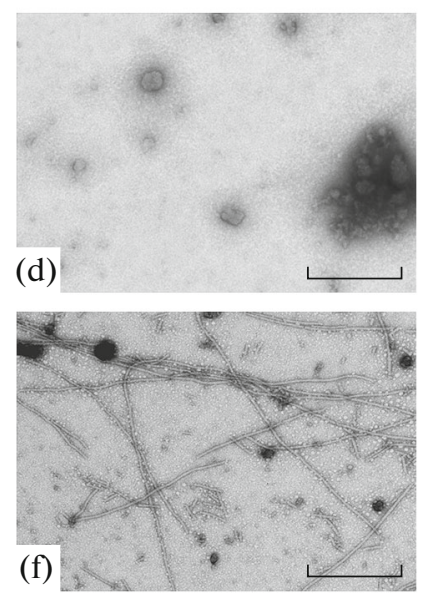

Fig. 3. Products of ER6 and AltMV CP incubation with viral RNA. (a) AltMV CP; (b) ER6; (c) AltMV RNA + AltMV CP (1: 7); (d, e) AltMV RNA + ER6 (1: 7); (f) AltMV RNA + AltMV CP + ER6 (1: $7: 7)$. Transmission electron microscopy with $2 \%$ uranyl acetate contrast. The proportion between viral RNA and proteins (AltMV $\mathrm{CP}$ and/or ER6) is indicated in parentheses. Scale bars: (a, c, e) $200 \mathrm{~nm}$, (b) $600 \mathrm{~nm}$, (d, f) $400 \mathrm{~nm}$.

along with the results of the gel retardation assay, invited a hypothesis that mosaic RNP assembly that involved not only native AltMV CP, but also ER6 occurred.

\section{ER6 within RNPs Retains Antigenic Specificity of Rotavirus $A$}

Antigenic specificity of ER6 within RNPs obtained by incubation of ER6 and AltMV CP with viral RNA was assessed by immunoblotting. For this purpose, RNPs were separated by electrophoresis in an agarose gel and transferred onto a membrane by wet transfer. RNPs obtained by incubation of RNA with a mixture of ER6 and native AltMV CP were specifically recognized by polyclonal antibodies to rotavirus A (Fig. 4, lane 1), which indicates that ER6 within RNPs retained the antigenic specificity of rotavirus A. In preparations obtained by incubation of RNA with ER6 only, the antibodies also detected the products of interaction (Fig. 4, lane 3). However, these products had a higher electrophoretic mobility than RNPs obtained by incubation of RNA with a mixture of two proteins. Antibodies to rotavirus A did not interact

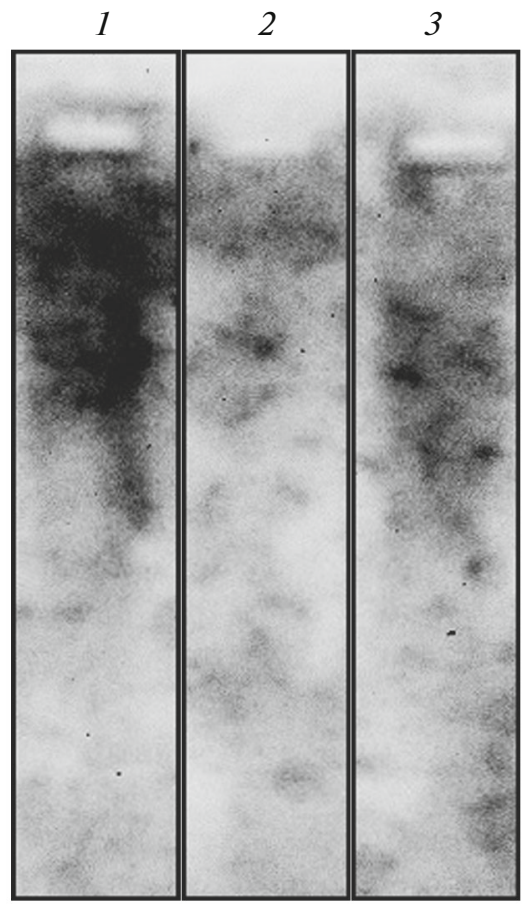

Fig. 4. ER6 within RNPs retains specificity as a rotavirus A antigen. Immunoblotting analysis: (1) AltMV RNA + AltMV CP + ER6 (1: $7: 7)$; (2) AltMV RNA + AltMV CP (1:7) (negative control); (3) AltMV RNA + ER6 (1: 7). Primary antibodies were commercial polyclonal antibodies to rotavirus A. Secondary antibodies were species-specific antibodies conjugated with horseradish peroxidase. The proportion between viral RNA and proteins (AltMV $\mathrm{CP}$ and/or ER6) is indicated in parentheses.

with the material loaded on lane 2 (AltMV CP + AltMV RNA).

\section{DISCUSSION}

In this work, we studied the possibility of constructing a rotavirus antigen based on the coat protein of AltMV, a plant virus, and the RV14 epitope of rotavirus A protein VP6 (amino acid residues 289-302, RLSFQLMRPPNMTP). This epitope was reported to induce a strong protective immune response, comparable to that observed for immunization with fullsize VP6, in mice [7]. This fragment is conserved in a number of group A strains [6] and can be considered a promising antigenic determinant for development of vaccines against rotavirus infection.

To enhance the immunogenicity of this epitope, we selected the coat protein of the plant virus AltMV (strain MU) as a carrier. According to the data obtained in our previous work, both AltMV virions and VLPs exhibit adjuvant properties [9], which inspired hope that VLPs or RNPs formed with AltMV $\mathrm{CP}$ could efficiently enhance the immune response to the epitope of rotavirus A protein VP6. Based on the study by Tremblay et al., who showed that in VLPs of 
the closely related papaya mosaic virus, the C-terminus of the coat protein is exposed on the outer surface, we supposed that the C-terminus of AltMV CP would also be exposed in the corresponding VLPs or RNPs and therefore the rotavirus epitope would be accessible for recognition by the immune system.

In this work, we designed a genetic construct that encodes a chimeric protein AltMV CP fused with an epitope of rotavirus VP6. It was demonstrated that the resulting recombinant protein ER6 was specifically recognized by commercial polyclonal antibodies to rotavirus $\mathrm{A}$ and, therefore can be utilized as a recombinant rotavirus A antigen.

We also examined the possibility of obtaining ER6containing RNPs. For this purpose, AltMV RNA was incubated with native AltMV CP (control), ER6, or with a mixture of both proteins. The proportions between the amounts of RNA and proteins (AltMV CP and/or ER6) used to obtain RNPs had been optimized in our previous study where RNPs of another potexvirus, potato virus $X$, were obtained [17, 18]. Using a gel retardation assay, it was found that RNPs of different molecular weights were formed in a mixture of AltMV RNA with AltMV CP and ER6. Subsequent analysis by electron microscopy confirmed the presence of particles of different lengths and morphology resembling AltMV virions. For the mixtures where AltMV RNA was incubated with each of the proteins individually, the gel retardation assay revealed that the material was held up at the start of the gel. Data from our previous experiments suggest that the presence of histidine residues can lead to nonspecific aggregation of recombinant proteins with RNA [20]. This may explain the observation that RNA incubated with recombinant protein ER6 was retained in the gel at the start level. Electron microscopy showed that under conditions of RNP formation, AltMV CP produced long filamentous VLPs, which is consistent with the previously published data [12]. Under the selected conditions, addition of RNA did not affect the degree of AltMV CP polymerization. It can be supposed that long filamentous VLPs mechanically prevented RNA from advancing in the gel. However, incubation of AltMV RNA with ER6 produced, along with aggregates, a certain number of structures with the morphology of VLP conglomerates (Fig. 3e), which were absent in the RNA-free ER6 specimen. These structures probably represent the products of specific interaction between recombinant ER6 and viral RNA. Therefore, it can be concluded that ER6 interacts with viral RNA but this interaction does not result in RNP formation. At the same time, incubation of AltMV RNA with both proteins simultaneously (ER6 and AltMV CP) gave rise to RNPs of different lengths and morphology resembling AltMV virions; apparently, they were generated by mosaic assembly. Interestingly, addition of ER6 to RNA and AltMV CP prevented the formation of extremely long AltMV VLPs. Analysis of RNPs obtained by incubation of ER6, AltMV CP, and
AltMV RNA using immunoblotting with polyclonal antibodies to rotavirus A confirmed that the resulting RNPs contained chimeric ER6, which retained its antigenic specificity within RNPs. ER6 aggregates with RNA also interacted with polyclonal antibodies to rotavirus A. Previously, we showed that AltMV and AltMV VLPs comparable to virions in size have a pronounced immunostimulating potential [11]. Therefore, it is RNPs obtained by interaction of RNA with both AltMV CP and ER6 that represent most potential for development of a vaccine prototype.

In this work we obtained RNPs composed of AltMV RNA, native AltMV CP, and recombinant ER6. It was shown that ER6 retained its antigenic specificity within these RNPs. Based on this promising result, it is expected that RNPs containing ER6 and other antigenic determinants of rotavirus might become a basis for development of a safe recombinant vaccine candidate against rotavirus infection.

\section{FUNDING}

This work was supported by the Russian Foundation for Basic Research (project no. 18-34-00006mol_a) and by the program "Fundamentals of Technology and the Use of the Features of Nanostructures in Life Sciences" of the Russian Academy of Sciences.

\section{COMPLIANCE WITH ETHICAL STANDARDS}

Conflict of interests. The authors declare that they have no conflict of interest.

Statement on the welfare of animals. This article does not contain any studies involving animals or human subjects performed by any of the authors.

\section{REFERENCES}

1. Tate J.E. Burton A.H., Boschi-Pinto C., Parashar U.D. 2016. Global, regional, and national estimates of rotavirus mortality in children $<5$ years of age, 2000-2013. Clin. Infect. Dis. 62 (Suppl. 2), S96-S105

2. Desselberger U., Wolleswinkel-van den Bosch J., Mrukowicz J., Rodrigo C., Giaquinto C., Vesikari T. 2006. Rotavirus types in Europe and their significance for vaccination. Pediatr. Infect. Dis. 25 (1), S30-S41.

3. Kondakova O.A., Nikitin N.A., Trifonova E.A., Atabekov J.G., Karpova O.V. 2017. Rotavirus vaccines: New strategies and approaches. Moscow Univ. Biol. Sci. Bull. 72 (4), 169-178.

4. Jiang B., Genstch J.R., Glass R.I. 2008. Inactivated rotavirus vaccines: A priority for accelerated vaccine development. Vaccine. 26 (52), 6754-6758.

5. Estes M.K., Greenberg H.B. 2013. Rotaviruses. In Fields Virology. Eds. Knipe D.M., Howley P. Philadelphia: Wolters Kluwer Health, pp. 1347-1395.

6. Parbhoo N., Dewar J.B., Gildenhuys S. 2016. Sequence analysis and structural implications of rotavirus capsid proteins. Acta Virol. 60 (3), 260-270. 
7. Choi A.H.C., Basu M., McNeal M.M., Flint J., VanCott J.L., Clements J.D., Ward R.L. 2000. Functional mapping of protective domains and epitopes in the rotavirus VP6 protein. J. Virol. 74 (24), 11574-11580.

8. Trifonova E.A., Zenin V.A., Nikitin N.A., Yurkova M.S., Ryabchevskaya E.M., Putlyaev E.V., Donchenko E.K., Kondakova O.A., Fedorov A.N., Atabekov J.G., Karpova O.V. 2017. Study of rubella candidate vaccine based on a structurally modified plant virus. Antiviral Res. 144 (C), 27-33.

9. Donchenko E.K., Trifonova E.A., Nikitin N.A., Atabekov J.G., Karpova O.V. 2018. Alternanthera mosaic potexvirus: Several features, properties, and application. Adv. Virol. 2018, Art. ID 1973705. https://doi.org/10.1155/2018/1973705

10. Donchenko E.K., Pechnikova E.V., Mishyna M.Yu., Manukhova T.I., Sokolova O.S., Nikitin N.A., Atabekov J.G., Karpova O.V. 2017. Structure and properties of virions and virus-like particles derived from the coat protein of Alternanthera mosaic virus. PLoS One. 12 (8), e0183824.

11. Petrova E.K., Trifonova E.A., Nikitin N.A., Karpova O.V. 2015. Adjuvant properties of Alternanthera mosaic virus virions and virus-like particles. FEBS J. 282 (Suppl. 1), 134.

12. Mukhamedzhanova A.A., Smirnov A.A., Arkhipenko M.V., Ivanov P.A., Chirkov S.N., Rodionova N.P., Karpova O.V., Atabekov J.G. 2011. Characterization of Alternanthera mosaic virus and its coat protein. Open Virol. J. 5, 136140.

13. Ivanov P.A., Mukhamedzhanova A.A., Smirnov A.A., Rodionova N.P., Karpova O.V., Atabekov J.G. 2011. The complete nucleotide sequence of Alternanthera mosaic virus infecting Portulaca grandiflora represents a new strain distinct from phlox isolates. Virus Genes. 42 (2), 268-271.
14. Sambrook J., Fritsch E.F., Maniatis T. 1989. Molecular Cloning: A Laboratory Manual, 2nd ed. Cold Spring Harbor, NY: Cold Spring Harbor Lab. Press.

15. Qiagen Inc. 2003. The QIAexpressionist: A Handbookfor High-level Expression and Purification of $6 \times$ His-Tagged Proteins, 5th ed. Valencia: Qiagen.

16. Nikitin N.A., Malinin A.S., Rakhnyanskaya A.A., Trifonova E.A., Karpova O.V., Yaroslavov A.A., Atabekov J.G. 2011. Use of a polycation spacer for noncovalent immobilization of albumin on thermally modified virus particles. Polym. Sci. Ser. A. 53 (11), 1026-1031.

17. Karpova O.V., Zayakina O.V., Arkhipenko M.V., Sheval E.V., Kiselyova O.I., Poljakov V.Yu., Yaminsky I.V., Rodionova N.P., Atabekov J.G. 2006. Potato virus X RNA-mediated assembly of single-tailed ternary 'coat protein-RNA-movement protein' complexes. J. Gen. Virol. 87, 2731-2740.

18. Karpova O.V., Arkhipenko M.V., Zayakina O.V., Nikitin N.A., Kiselyova O.I., Kozlovsky S.V., Rodionova N.P., Atabekov J.G. 2006. Regulation of RNA translation in potato virus X RNA-coat protein complexes: The key role of the N-terminal segment of the protein. Mol. Biol. (Moscow). 40 (4), 628-634.

19. Tremblay M.H., Majeau N., Gagné M.E.L., Lecours K., Morin H., Jean-Baptiste Duvignaud J.B., Bolduc M., Chouinard N., Paré C., Gagné S., Leclerc D. 2006. Effect of mutations K97A and E128A on RNA binding and self-assembly of papaya mosaic potexvirus coat protein. FEBS J. 273 (1), 14-25.

20. Zayakina O., Arkhipenko M., Smirnov A., Rodionova N., Karpova O., Atabekov J. 2009. Restoration of potato virus X coat protein capacity for assembly with RNA after His-tag removal. Arch. Virol. 154 (2), 337-341.

Translated by D. Timchenko 